\title{
Simulation and analysis of improved direct torque control of switched reluctance machine
}

\author{
Alexander Krasovsky \\ Department of Electrical Engineering and Power Electronics, Bauman Moscow State Technical University (BMSTU), \\ Russian Federation
}

\begin{tabular}{l} 
Article Info \\
\hline Article history: \\
Received Sep 5, 2019 \\
Revised Nov 12, 2019 \\
Accepted Nov 26, 2019 \\
\hline
\end{tabular}

Keywords:

Direct torque control Motoring and braking modes Simulation model Simulation results Switched reluctance machine

\begin{abstract}
Direct torque control of electric machines widely used in modern electric drives. Switched reluctance machines (SRM) are different from traditional electric machines, so we cannot apply well-known technical solutions to them. Simulation can provide a powerful approach for investigating the control of switched reluctance machines, and Matlab/Simulink allows it successfully implemented. This paper presents a description of the model and the simulation results of the proposed new algorithms for direct control of the instantaneous torque of SRM in the motor and braking modes. In comparison with the known control algorithms, the proposed algorithm uses one common for all phases relay regulator with a smaller number of switching thresholds and, therefore, it has greater reliability and is easier to set up.
\end{abstract}

Copyright $₫ 2020$ Institute of Advanced Engineering and Science. All rights reserved.

\section{Corresponding Author:}

Alexander Krasovsky,

Department of Electrical Engineering and Power Electronics,

Bauman Moscow State Technical University,

Moscow, Russian Federation

Email: kralb64@gmail.com

\section{INTRODUCTION}

Switched reluctance machines (SRM) attract the interest of many experts because of their constructive simplicity and wide control resources. [1-3]. The operation of the SRM consists of discrete switching of the phase windings, therefore, with usual control algorithms, significant ripple of instantaneous torque appear in them. The most severe torque ripple occurs when current and torque shift from one to another phase [4]. There are various approaches reducing the ripples of the SRM torque. The approaches based on phase current profiling, on so-called torque sharing functions (TSF) and on direct instantaneous torque control (DITC) are the most popular ones among them [5-14]. Their basis is the formation of certain patterns of change in phase currents and torques of the SRM on the interval of their switching. The main differences between them consist of the method of determining these laws and in their technical implementation. TSF is one of the best-known approaches to determining the laws of variation of the SRM phase currents in the phase switching interval [8-11]. The main problem in the practical implementation of TSF is the absence of an unambiguous method for determining specific components of the torque and currents of the phases to minimize torque ripples.

The most detailed and comprehensively physical causes of the occurrence of pulsations of the torque of SRM and the possibility of their complete elimination by means of control were considered in [12] with the participation and under the guidance of the author of this work. In [12] we also proposed and substantiated the most rational in our opinion the algorithm for automatic forming of the voltage on the switched phases in the following sequence. The full positive voltage of the power supply $+U_{\mathrm{DC}}$ is applied to the switched-on phase, and the voltage on the switched-off phase is gradually reduced to its minimum negative value $-U_{\mathrm{DC}}$ in order to satisfy the condition of constant total torque. Then the voltage at the 
disconnected phase fixed at the level $-U_{\mathrm{DC}}$ and the voltage at the switched-on phase start to reduce from the condition of ensuring the constancy of the total torque. Since the voltage at one of the switched phases is always equal to plus or minus $U_{\mathrm{DC}}$, such a control ensures phase switching at the shortest possible interval, which ensures maximum speed and minimization of switching losses. At the same time, such control allows minimizing the requirements for the voltage level $U_{\mathrm{DC}}$. According to our information, a similar algorithm in English described only after 13 years in [13].

Obviously, from the standpoint of achieving the maximum speed of the electric drive while compensating for the pulsations of the torque, our proposals and the proposals of the authors of the work, which we obviously obtained independently of each other, are the most effective in the context of the problem under consideration. Next, author considers the most well-known implementations of the algorithm for automatic changing the voltage on the switched phases with most progressive to date direct torque control of the SRM and offer its variant with improved characteristics for motor and braking operation modes this machine.

\section{CONVENTIONAL ALGORITHMS OF DIRECT INSTANTANEOUS TORQUE CONTROL OF SRM IN MOTORING MODE}

DITC is similar to the well-known vector approach to controlling an induction motor [14]. Among DITC of SRM, only control of instantaneous torque and control simultaneous instantaneous torque and motor flux are distinguished. The second one has the best ability to reduce noise and vibration, but leads to increased values of the current consumed from the power source, which can be a significant limitation, for example, in SRM for traction applications [15]. This article is devoted to the development of the direct control method only of the instantaneous SRM torque. Figure 1 show a typical structure of the direct torque control system for the m-phase SRM [14]. It includes the bloc for estimating the instantaneous value of the total torque $T_{\square}$ by its components $T_{\mathrm{ph} 1 \ldots \mathrm{m}}$ from each motor phase switched at the moment and the relay digital torque regulator. This regulator generates the control signals $S_{1 \ldots \mathrm{m}}$ to the corresponding switched elements of the inverter as a function $\square T_{\square}$, i.e of deviation of torque $T_{\square}$ from the specified values $T_{\text {ref. The instantaneous }}$ values of the torque $T_{\mathrm{ph}, 1 \ldots \mathrm{m}}$ are usually estimated by the measured values of the phase currents $I_{\mathrm{ph} 1 \ldots \mathrm{m}}$ taking into account the nonlinear characteristic $T_{\mathrm{ph}}\left(I_{\mathrm{ph}}, \Theta\right)$, where $\Theta$ is the angular misalignment of the stator and the rotor poles.

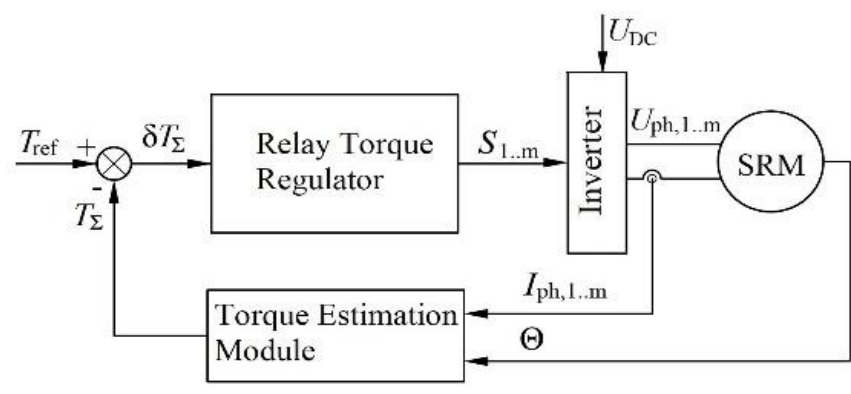

Figure 1. The SRM direct instantaneous torque control system structure

Of the well-known publications dealing with DITC of the SRM the articles, $[14,16]$ deserves special attention. In [14], the transmission of the torque control from one phase to another occurs due to a special choice of the hysteresis zones of the corresponding relay elements, but the rationale for its selection is not given. The oscillogram in [14] shows that in a significant interval of operation the switched SRM phases are in a short-circuited state, and therefore the rate of current change in them is small. This does not allow the power supply full use in the formation of the specified torque value $T_{\square}$. In [16] also proposed an algorithm for controlling the instantaneous torque of SRM. The authors used torque hysteresis regulators for each individual phase, and each one works independently. Considered a hysteresis regulator with two outputs states $(\mathrm{S}=1$ and $\mathrm{S}=-1)$ and three outgoing states $(\mathrm{S}=1, \mathrm{~S}=0$ and $\mathrm{S}=-1)$. Outputs state $\mathrm{S}=1$ corresponds to connecting the phase to the positive voltage of the power source $+U_{\mathrm{DC}}, \mathrm{S}=0$ corresponds to a shorted phase when the voltage on it is zero and $S=-1$ corresponds to connecting the phase to the negative voltage of the power source $-U_{\mathrm{DC}}$. Torque of the switched-on $T_{\text {on }}$ and the switched-off $T_{\text {off }}$ phases formed during the switching interval with maximum use of the power supply voltage $U_{\mathrm{DC}}$. 
As follows from [16], a simpler, two-state hysteresis regulator should have four different switching thresholds. However, it provides an increased switching frequency causing an additional increase in losses. When using the three-state hysteresis regulator, the switching frequency of the switched elements decreases. Such a regulator has six different thresholds for the operation of the relay element (Figure 7 in [16]). Setting up such a regulator is quite complicated and in the process of its operation, there is a high probability of failures due to various interferences. In addition, the authors do not consider the adaptation of this controller to the SRM braking mode in this article. Since this mode has its own specifics, in an unchanged form this regulator cannot be used in the braking mode of SRM. This significantly limits its capabilities, especially in regulated highly dynamic electric drives. For research and development of new SRM control algorithms, simulation is best suited. To evaluate the effectiveness of the proposed DITC algorithm, we used a simulation model of an SRM-based electric drive in MATLAB-SIMULINK environment [17, 18].

\section{DESCRIPTION OF THE SIMULATION MODEL}

The general principle of simulation models the creation of SRM and its feature explicitly described in $[19,20]$. The use of Miller's curves in models is the rarest in the literature, so we will pay more attention to this in this article. T. J. E. Miller found [21, 22] that if the magnetization curves of a phase SRM are represented as $\Psi(\Theta)$ dependences for a number of phase current values $I_{\mathrm{ph}}$ (as an example, Figure. 2 (a) shows curves for four current values $I_{1}<I_{2}<I_{3}<I_{4}$ ), and then normalize them using the expressions

$$
\begin{aligned}
& y(x)=\frac{\Psi(x)-\Psi_{\text {min }}}{\Psi_{\text {max }}-\Psi_{\text {min }}} ; \\
& x=\frac{\Theta-\Theta_{\text {min }}}{\Theta_{\text {max }}-\Theta_{\text {min }}},
\end{aligned}
$$

then the obtained dependences $y(x)$ for different values of current $I_{\mathrm{ph}}$ almost coincide. Here $\Psi_{\max }-$ flux linkage in the aligned position of the rotor and stator $\Theta_{\max } ; \Psi_{\min }$ - flux linkage in the unaligned position of the rotor and stator $\Theta_{\min } ; \Psi(x)$ - flux linkage in the intermediate position of the rotor and stator $x$. Note that in expression (1), the flux linkages $\Psi(x), \Psi_{\max }, \Psi_{\min }$ are determined for the same current value $I_{\mathrm{ph}}$.

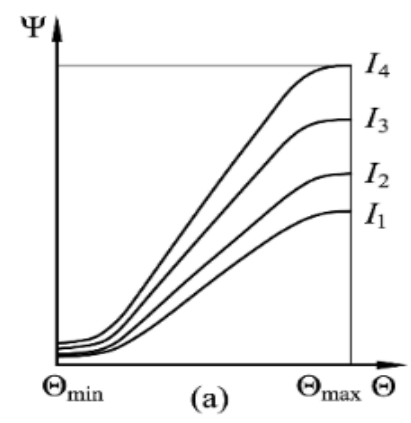

(a)

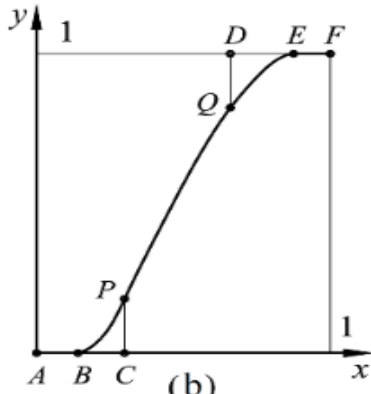

(b)

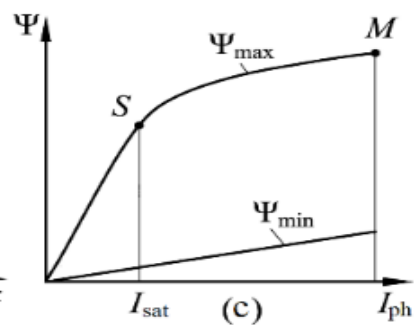

(c)

Figure 2. Dependencies $\Psi(\Theta)$ (a), normalized (b) and $\Psi\left(I_{\mathrm{ph}}\right)$ in the mismatched and coordinated positions of the rotor SRM (c)

The averaged dependencies $y(x)$ allow establishing the shape of its magnetic characteristics in the intermediate positions of the rotor using the known magnetization curves in aligned and unaligned positions. T. J. E. Miller also proposed a variant of the analytical approximation of the magnetization curves in the aligned and unaligned rotor positions, and the normalized curve $y(x)$. Figures 2 (b) and (c) show the characteristic appearance of these curves. The dependence $\Psi_{\min }\left(I_{\mathrm{ph}}\right)$ is assumed linear in the entire range of variation of the phase current $I_{\mathrm{ph}}$, i.e.

$$
\Psi_{\text {min }}\left(I_{\mathrm{ph}}\right)=I_{\mathrm{ph}} L_{\text {min }}
$$


where $L_{\min }$ is the phase inductance in the unaligned position of the rotor. The curve $\Psi_{\max }\left(I_{\mathrm{ph}}\right)$ consists of two sections. To the left of the point $S \square$ for $I_{\mathrm{ph}}<I_{\mathrm{sat}}$, the dependence is also approximated by a linear function

$$
\Psi_{\max }\left(I_{\mathrm{ph}}\right)=I_{\mathrm{ph}} L_{\max }
$$

where $L_{\max }$ is the phase inductance in the aligned position of the rotor, $I_{\text {sat }}$ - current start of saturation of the magnetic system of the SRM. A parabolic function approximates a curve in a nonlinear segment from point $\mathrm{S}$ to point $\mathrm{M}$

$$
\left(\Psi_{\max }-\Psi_{\mathrm{sat0}}\right)^{2}=4 a\left(I_{\mathrm{ph}}-I_{\mathrm{sat}}\right)
$$

where $I_{\text {sat0 }}=I_{\text {sat }}-\frac{a}{L_{\mathrm{pac}}^{2}} ; \Psi_{\mathrm{sat} 0}=\Psi_{\mathrm{sat}}-\frac{2 a}{L_{\mathrm{max}}} ; a=\frac{\Delta \Psi^{2}}{4\left(\Delta I-\Delta \Psi / L_{\text {max }}\right)} ; \Delta \Psi=\Psi_{\mathrm{M}}-\Psi_{\mathrm{S}} ; \Delta I=I_{\mathrm{M}}-I_{\text {sat }}$.

The normalized curve $y(x)$, as shown in Figure 2 (b), according to [21] consists of five characteristic zones. The phase current $I_{\mathrm{ph}}$ is determined from the solution of (1) by substituting the expressions for $\square_{\text {min }}$ ( $\left.I_{\mathrm{ph}}\right)$ from (3) and $\square \max \left(I_{\mathrm{ph}}\right)$ from (4) with $I_{\mathrm{ph}}<I_{\mathrm{sat}}$ and from (5) with $I_{\mathrm{ph}}>I_{\mathrm{sat}}$. The general expression for the phase torque $T_{\mathrm{ph}}$, taking into account the accepted notation, takes the form:

$$
T_{\mathrm{ph}}=\frac{1}{\Theta_{\max }-\Theta_{\min }} \frac{d y(x)}{d x} \cdot \int_{0}^{I_{\mathrm{ph}}}\left(\Psi_{\max }-\Psi_{\min }\right) d I_{\mathrm{ph}}
$$

Using the above expressions, we implemented the simulation model of a single-phase SRM, as shown in Figure 3. In this model, the subsystem CM calculates the values $\square_{\max }\left(I_{\mathrm{ph}}\right)$ and $\square_{\min }\left(I_{\mathrm{ph}}\right)$, the subsystem NFl $-y(x)$. In the Algebraic Constraint block, at each instant of time, the value of the phase current $I_{\mathrm{ph}}$ is determined at which equality (1) is fulfilled. The NFl subsystem also calculates values depending on the current angle value, and the Product 2 block calculates the torque of phase $T_{\mathrm{ph}}$.

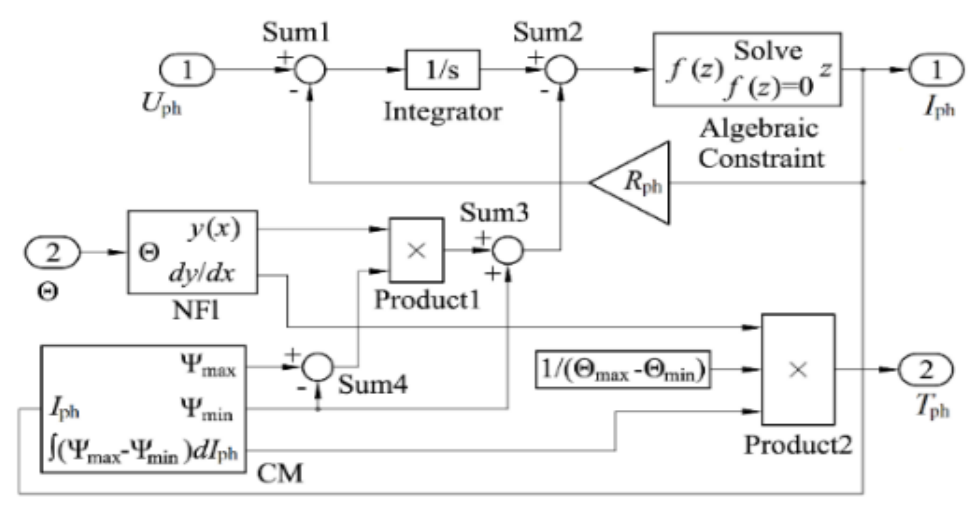

Figure 3. Simulation model of phase SRM based on "Miller's curves

Figure 4 (a) and (b) shown information about the accuracy of calculations. Dashed blue lines on Figure 4 (a) show a number of magnetic characteristics of the four-phase SRM with typical parameters and nominal output power $5 \mathrm{~kW}$ obtained as a result of the inverse transformation of the analytical representation of the $\Psi_{\min }\left(I_{\mathrm{ph}}\right)$ and $\Psi_{\max }\left(I_{\mathrm{ph}}\right)$ dependencies in accordance with (3), (4), (5) and the "normalized Miller's curve".

These dependencies we used in the model. In the same figure, the solid lines represent the family of experimental magnetic characteristics $\Psi\left(I_{\mathrm{ph}}, \Theta\right)$ the same SRM. As can be seen in Figure 4 (a), the calculated dependences are quite close to the experimental characteristics taken under the same conditions, and they practically merge in the zone of local saturation of the SRM poles, and the largest discrepancy between them is observed in the zone of greatest curvature of each curve $\Psi\left(I_{\mathrm{ph}}\right)$. The Figure 4 (a) shows that the maximum error is about $2.5 \%$. The torque curves $T_{\mathrm{ph}}(\Theta)$ in Figure 4 (b) are static characteristics obtained at fixed 
positions of the shaft SRM when the phases are supplied with direct current. In contrast to the experimental characteristics, the calculated curves $T_{\mathrm{ph}}(\Theta)$ have fractures in the zone of a consistent pole position $\Theta=45^{\circ}$. However, the error resulting from this is small, which allows to concluding that the model is reasonably well adapted to the study of issues of control of SRM torque.

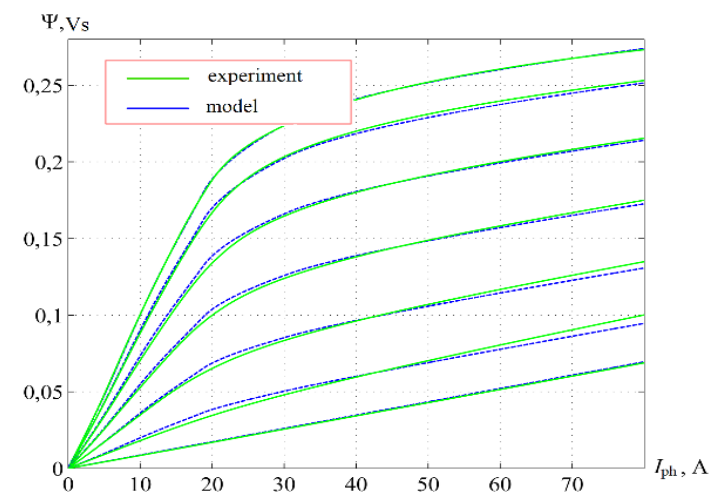

(a)

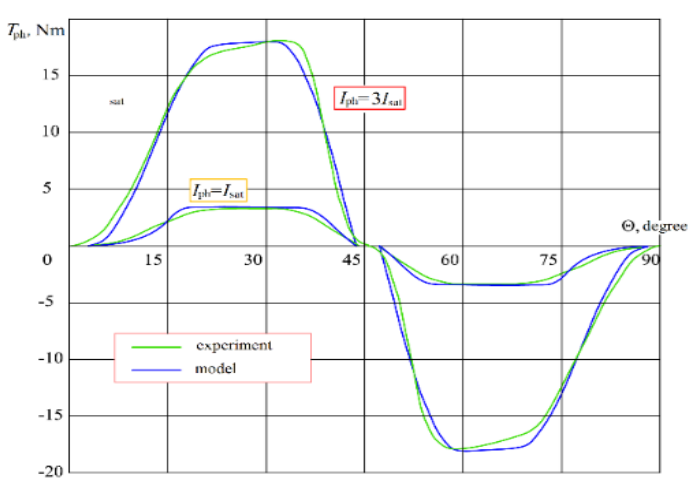

(b)

Figure 4. Family of $\square\left(I_{\mathrm{ph}}\right)$ characteristics obtained as a result of the "Miller's transformation" with experimental dependences (a) and comparison of calculated and experimental of torque curves $T_{\mathrm{ph}}(\square)$ (b)

\section{SIMULATION OF THE PROPOSED DIRECT INSTANTANEOUS TORQUE CONTROL IN MOTOR MODE OF SRM}

The general block diagram of our proposed novel DITC for SRM is similar to that shown in Figure 1. We propose to use one common hysteresis regulator to control currents of all phases and making the hysteresis regulator itself simpler and therefore more reliable and facilitate its adjustment. Since the voltage regulation alternately performed only in one of the phases, while on other phases, the voltage is maintained, or at the level $+U_{\mathrm{DC}}$ or $-U_{\mathrm{DC}}$, or phase voltage is zero, there is no need to use an individual regulator for each of phase. The sequence of the transition of regulation from one phase to another we propose to perform using a special logic switch. Functional diagram of the switching control system of two adjacent phases SRM shown in Figure 5(a). Voltage and current profiles of two adjacent phases of SRM to explain the principle of the new DITC shown in Figure 5(b). As shown in Figure 5(a), the control system includes a special unit Calculator for calculating the switching on positions for each phase of the SRM $\Theta_{\text {on1 }}$, $\Theta_{\mathrm{on} 2}$ etc. For calculations, the values of the speed $\square$, voltage of the power source $U_{\mathrm{DC}}$ and the set value of the total torque $T_{\square \square}$ are used, taking into account the electromagnetic parameters of the motor [17, 18].

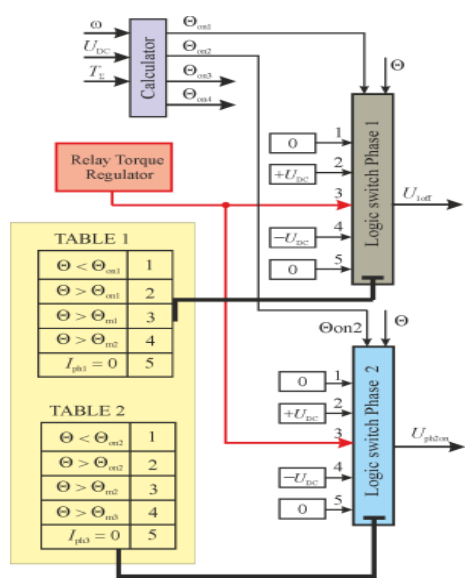

(a)

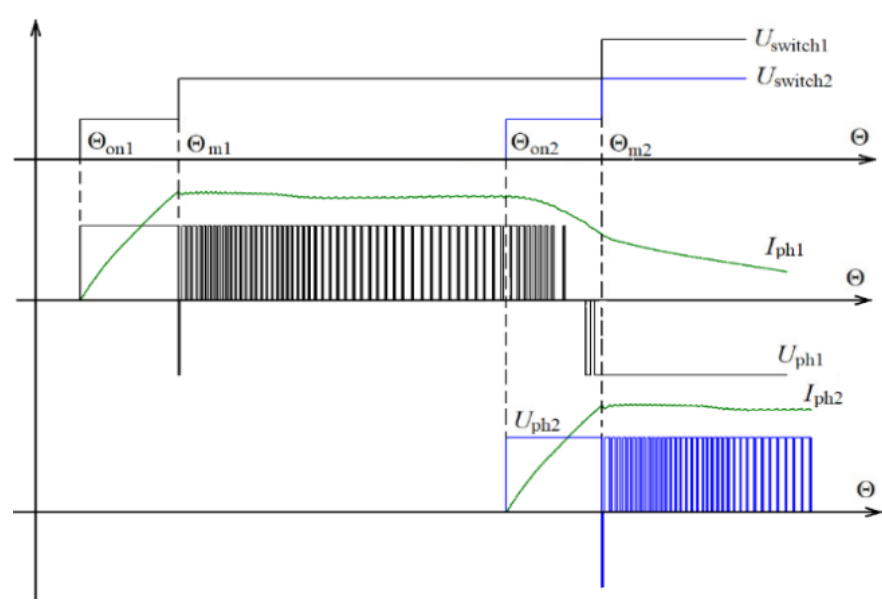

(b)

Figure 5. Functional diagram of the switching control system of two adjacent phases SRM (a) and their profiles of the voltage and current (b)

Simulation and analysis of improved direct torque control of switched reluctance... (Alexander Krasovsky) 
Special multiport switch (in Figure 5(a) we have shown only two its units for phase 1 and phase 2) carry out the successive change of the laws of voltage regulation at phases 1 and 2 as a function of the rotor position $\Theta$. Each unit chooses among several inputs at 1 to 5 and transmits one of them to output. Each unit has a control input and 5-date input. The change of control signals $U_{\text {switch1 }}$ and $U_{\text {switch2 }}$ for these blocks shown in the upper part of Figure 5(a). It is important to emphasize that all switching positions, except the switching on position of each phase, do not need to be set in advance, they are determined automatically. When the condition $\square<\Theta_{\text {on } 1}$ is fulfilled for the phase 1 and $\square<\Theta_{\text {on2 }}$ for the phase 2, the voltages on these phases $U_{\text {ph1 }}$, $U_{\mathrm{ph} 2}$ and currents $I_{\mathrm{ph} 1}, I_{\mathrm{ph} 2}$ are zero. Phase 1 is energized voltage $+U_{\mathrm{DC}}$ at $\square=\Theta_{\mathrm{on} 1}$ and it is maintained at this level to the position $\Theta_{\mathrm{m} 1}$. Phase 2 is energized voltage $+U_{\mathrm{DC}}$ at $\square=\Theta_{\mathrm{on} 2}$ and it is maintained at this level to the position $\Theta_{\mathrm{m} 2}$. As we see from Figure 5(b), positions $\Theta_{\mathrm{m} 1}$ and $\Theta_{\mathrm{m} 2}$ correspond to the condition of the beginning of the pulse regulation voltages $U_{\mathrm{ph} 1}, U_{\mathrm{ph} 2}$ and the currents $I_{\mathrm{ph} 1}, I_{\mathrm{ph} 2}$ of the phase 1 and phase 2 . In this position, the voltage in the previous phase reaches the maximum negative value and its impulse regulation stops. These positions are automatically determined in the control system when the total torque $T_{\square} \square$ is outside the allowable limits of its change, which are set by the upper switching pitch of the relay element after the positions $\Theta_{\mathrm{on} 1}$ and $\Theta_{\mathrm{on} 2}$, respectively. As can be seen from Figure 5(b), starting from the position $\Theta_{\mathrm{m} 2}$ on the disconnected phase 1, the maximum negative voltage $-U_{\mathrm{DC}}$ maintained and the current in it drops off forcefully. As soon as the current of the disconnected phase drops to zero, this phase is disconnected from the voltage source and the voltage on it switches to zero.

Now let us take a closer look at the process of pulse voltage and current regulation of each phase. To regulate the SRM total torque $T_{\square}$, we propose to use an algorithm for changing the phase voltage in the form $U_{\mathrm{ph}}=U_{\mathrm{DC}} \operatorname{sign}\left(\square T_{\square}\right)$. To reduce the switching frequency of the inverter's switched element, a hysteresis zone with a width $\square_{\mathrm{M}}$ imposed into the switching algorithm. In addition, the third state is used when the SRM phase is short-circuited, i.e. $U_{\mathrm{ph}}=0$. Thus, the static characteristic of the torque relay controller in the motoring mode of SRM has the form, as shown in Figure 6(a), where $\square_{T}$ is the width of the hysteresis zone of relay element.

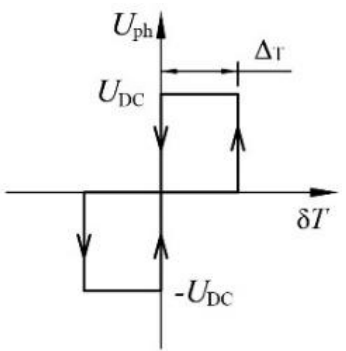

(a)

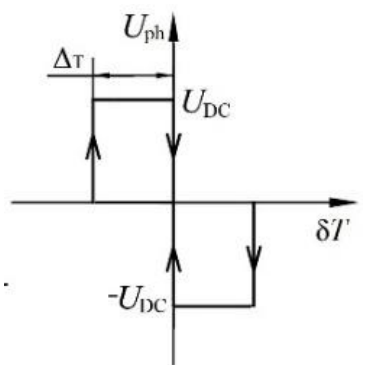

(b)

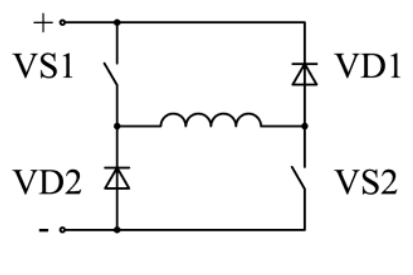

(c)

Figure 6. Torque regulator switching characteristic: in motoring mode (a), braking mode (b) and typical scheme of phase invertor (c)

In Figure 7(a) shows the change in the basic values of SRM when switching two adjacent phases.

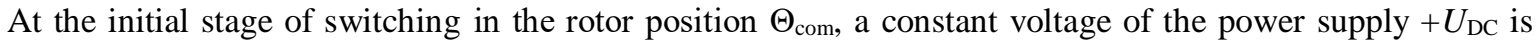
applied to the switched phase. The total torque $T_{\square}$ is regulated due to the corresponding effect on the voltage of the phase to be switched off $U_{\text {off. }}$ When the disconnected phase is no longer able to maintain the torque at a given level (angular position $\Theta_{\mathrm{m}}$ in Figure 7 (a)), the voltage on it is fixed at $-U_{\mathrm{DC}}$, and the torque is

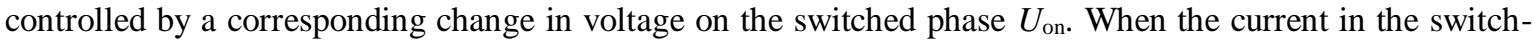
off phase decreases to zero, the moment is determined by the moment of the switch-on phase. Next, the cycle repeats. As can be seen, at any moment of time, regulation occurs only in one of the phases, and a constant voltage with an amplitude $U_{\mathrm{DC}}$ is applied to the second phase, which indicates the full use of the power supply by voltage.

As an example, an algorithm for the switched element operation of one phase of the inverter is given in accordance with its typical scheme (Figure 6(c)). It is supposed that in the initial state of the torque $T_{\square \square}$ is within the limits $\left(T_{\text {ref }}-\square_{T} ; T_{\text {ref }}\right)$, both inverters switched element VS1 and VS2 are closed and under the action of the voltage $U_{\mathrm{DC}}$, the phase current $I_{\mathrm{ph}}$ increases, which leads to the phase $T_{\mathrm{ph}}$ and the total torque

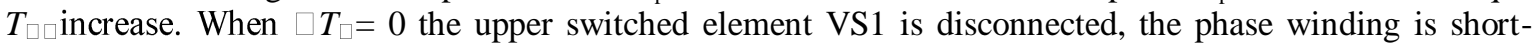
circuited and the current $I_{\mathrm{ph}}$, supported by the motion EMF, is reduced. The torques $T_{\mathrm{ph}}$ and $T_{\square}$ also decrease. 
The switching element VS1 will turn on again when the torque deviation $T_{\square}$ reaches $\square T_{\square}=-\square_{\mathrm{T}}$ from the set value. The previous state of the switched element retains in the range $\left(0 ; \square \square_{\mathrm{T}}\right)$.

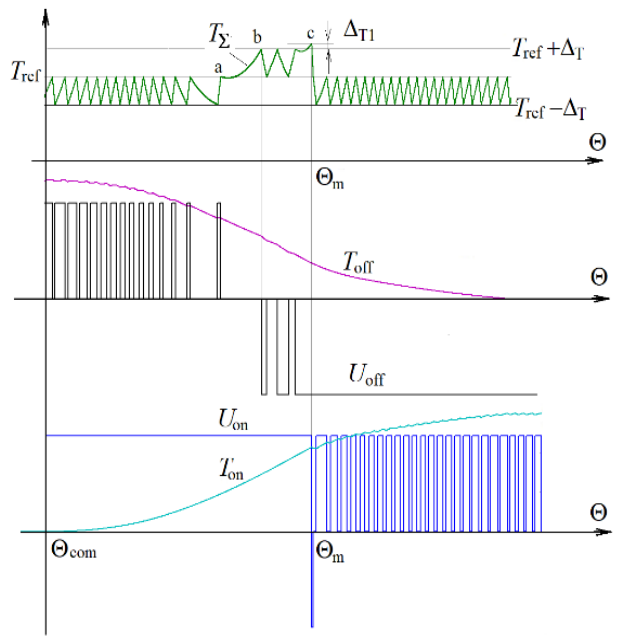

(a)

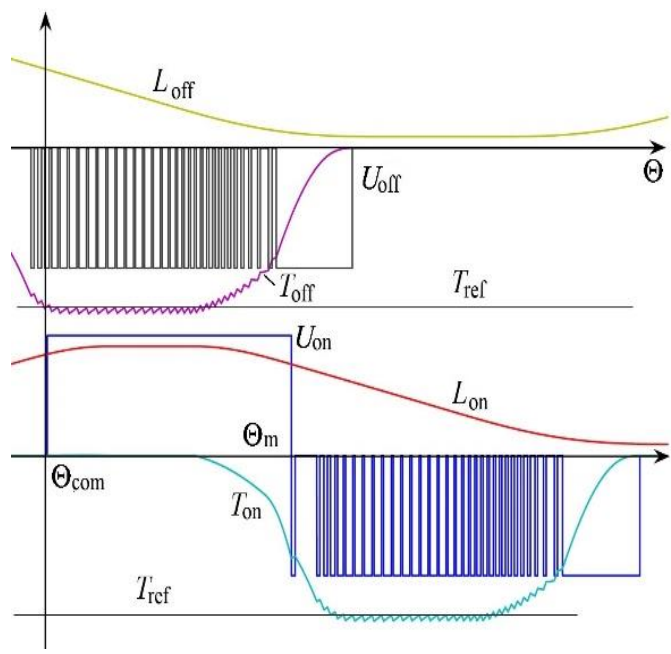

(b)

Figure 7. Modification of the torques and voltages on the switched-off and switched-on phases in motor (a) and braking (b) modes

When the switched elements VS1 and VS2 are turned off, the maximum negative voltage $-U_{\mathrm{DC}}$ is applied to the phase to be switched off, at the same time, the maximum positive voltage $U_{\mathrm{DC}}$ is applied to the switched-on phase. If the rate of increase in the torque of the switched-on phase is too high, the total torque goes beyond the upper limit of the hysteresis of the relay element (point $c$ in upper part of Figure 4). When setting up the control system, we set the allowable excess $\square_{\mathrm{T} 1}$ of the total torque $T_{\square} \square$ relative to ( $T_{\text {ref }}+$ $\square_{\mathrm{T}}$ ) and when it is reached, an additional threshold switch is triggered. We use its signal to control the logic switch (see Figure 5 (a), which gives a command to fix the voltage on the switched-off phase at the level $U_{\mathrm{DC}}$ and to the beginning of the voltage regulation on the switched-on phase. This phase has exhausted the control possibilities and control is transferred to the adjacent phase (position $\Theta_{\mathrm{m}}$ in Figure 7). Thus, in the proposed algorithm, it is possible to maintain the specified torque value $T_{\square} \square$ when using one common to all phases relay controller with a simple switching characteristic as shown in Figure 6, a and one common additional threshold device (or sensitive element) without hysteresis.

\section{FEATURES DITC OF SRM IN BREAKING MODE}

To control of the SRM in the braking mode in each commutation cycle, it is necessary to generate control actions that are "inverse" to the motor mode [18-19]. For example, if the state of the upper switched element in Figure 6(c) changes when the lower switched element is constantly turned on in the motor mode, then it will be necessary to change the state of either the upper or the lower switched element with the constantly closed opposite switched element in the braking mode. Thus, the winding shorted in the braking mode, the current flows through the diodes under the action of the EMF of the movement exceeding the supply voltage, when the VS1 switched element turned on and VS2 is off.

The static characteristic of the relay regulator in the braking mode is a mirror image of the characteristic in the motor mode (Figure 6(b)). At the stage operation of the phase, the adjustable value lies within the lower zone of the hysteresis. The upper switched element VS1 turns on and the current increases when $\square T_{\square}=0$. The upper switched element VS1 is disconnected, a negative voltage is applied to the winding, the current is reduced when $\square T_{\square}=\square \mathrm{T}$. The previous state of the switched elements retains in the range $(0 ; \square \square \mathrm{T})$. The second zone intended for SRM operation during the phase over commutation. The lower switched element VS2 closes, a positive voltage applied to the switched-on phase and the current and phase torque begin to increase forcefully. If the absolute value of the total torque continues to drop with a shorted winding, i.e. the absolute value of the switched-off phase torque decreases more rapidly than increases in the switched-on phase and reaches a $\left(T_{\text {ref }}-\square_{\mathrm{T}}\right)$ value. An uncontrolled current increase above permissible values may occur at the operation stage of the braking mode over commutation cycle at high speed. 
To explain this phenomenon, let us pay attention to the following. The speed increase at a constant voltage in the SRM motor mode results in a natural decrease of the steady-state current. In the braking mode, increasing the speed increases the current. At high speeds, the power supply voltage is not sufficient for rapid phase disconnection. As a result, even when the total negative power supply voltage applied to the winding, the current begins to increase until the phase inductance $L$ drops to minimum value $L_{\text {min. }}$ Such a mode is dangerous for power switched element and in order to avoid it, it is necessary to switch-on the phase later, so that during the switching-on phase the current increased to a value at which the current would not have exceeded the permissible value during operation. However, it will not be possible to eliminate the torque pulsation.

In Figure 7(b) shown the oscillogram of the SRM operation with DITC in the braking mode. At the initial stage of commutation in the $\Theta_{\text {com }}$ rotor position, an unchanged voltage of the power supply $+U_{\mathrm{DC}}$ is imposed to the switched-on phase. The instantaneous total torque controlled by the corresponding influence

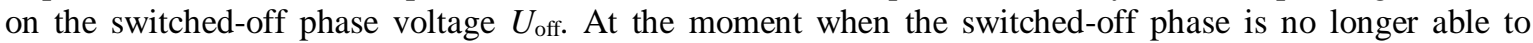
maintain the torque $T_{\square}$ at a given level (angular position $\Theta_{\mathrm{m}}$ at Figure 7(b)), the voltage on it is fixed at the $U_{\mathrm{DC}}$ level, and the torque control is carried out due to a corresponding change in the voltage $U_{\text {on }}$ on the switched-on phase. When the current in the switched-off phase reduces to zero, the torque $T_{\square}$ is determined by the torque of the switched-on phase. Then the cycle repeats. As seen, at any time the regulation occurs only in one of the phases, while the unchanged voltage of $U_{\mathrm{DC}}$ amplitude is impressed to the second phase that indicates the full use voltage of the power supply.

\section{ANALYSIS REZULTS AND DISCUSSION}

The following results are the most important results of the research. To create a torque $T_{\square}$ at the level of the average value $T_{\mathrm{av}}$ developed by the SRM with the traditional rectangular phase voltage form without torque pulsation compensation and single-phase switching, an additional phase voltage increase is required for DITC. For SRM with typical parameters, this increase is about 15-20\%. In the absence of such an increase near the beginning point of the SRM teeth overlapping, a torque control error can occur due to the simultaneous limitation of both $U_{\text {on }}$ and $U_{\text {off }}$ at the $U_{\mathrm{DC}}$ level for the following reasons:

a) Normally, when controlling the SRM, it is tended to maintain a current $I_{\mathrm{ph}}=$ const in most of the overlap zone of the stator and rotor teeth $[17,18]$. It is necessary to fulfill the condition $U_{\mathrm{ph}}=$ const in the first approximation. In this case, the average torque $T_{\text {av }}$ exceeds the torque developed by one phase in this interval $T_{\text {work }}$ due to a surge in the torque at the commutation stage (provided the angular zones overlapping of creating the motor torque at the level of the nominal torque by adjacent phases which is necessary for direct torque control). ). Therefore, if the torque $T_{\square}$ were equal to the torque $T_{\text {work }}$ at the stage of operation of one phase during the torque control at the phase commutation, it would be necessary to raise $T_{\text {work }}$ to the $T_{\text {av }}$ level, which would lead to the need of an additional increase of the current $I_{\phi}$ and the voltage $U_{\mathrm{ph}}$.

b) The switched-on phase develops a smaller torque $T_{\text {work }}$ even after the poles enter the overlap zone in order to compensate for the torque of the switched-off phase. As a result, when the torque of switchedoff phase due to the decrease in the derivative $(d L / d \Theta)$ off begins to drop rapidly, the voltage level $U_{\text {ph }}$ becomes insufficient to create the necessary rate rise of current and torque of switched-on phase. This is because the derivative $(d L / d \Theta)$ on has already reached its maximum and the motion EMF too high (the torque and the motion EMF of the phase are directly proportional to the value of the derivative $(d L / d \Theta)$.

The simulation showed that the proposed DITC algorithm has advantages over the algorithm proposed in [14], in which the computation unit of commutation angles works independently from the relay torque controller. In algorithm proposed in [14], a later or premature phase outage can occur. The running phase is turned off before the next one is able to maintain the cumulative torque at a given level (because of the small value of the derivative $d L / d \Theta$. Such a situation can arise if the torque creation zones at the nominal torque level in adjacent phases do not overlap. In the proposed DITC algorithm, the total torque continues to maintain at the expense of the switched-off phase torque until the torque reaches the nominal value in the switched-on phase (a slight surge of current can appear at the end of the commutation cycle).

\section{CONCLUSION}

The obtained simulation results on the Simulink / Matlab model fully confirm the performance and advantages of the proposed DITC of SRM in motor and brake modes. The author sees the main results of the research in the following: 
a) The proposed novel variant DITC of SRM allows to ensuring the maximum rate of change of the torque of adjacent phases in the interval of their switching when using only one common relay torque regulator. In comparison with the known control algorithms, the algorithm DITC proposed by us uses relay regulator with a smaller number of switching thresholds and, therefore, it has greater reliability and easier to set up. The degree of SRM torque ripple compensation at DITC depends on the accuracy of the representation of the phase torque characteristic $T_{\mathrm{ph}}\left(I_{\mathrm{ph}}, \square\right)$ used in the control algorithm and on the voltage of the power source.

b) An additional $15-20 \%$ increase in the power supply voltage is required for direct torque control to maintain the torque at the same level as with conventional SRM control.

c) The operation of the direct torque regulator in the SRM braking mode is impractical in the high-speed zone, because at the operating phase of the each phase commutation cycle an uncontrolled increase in the phase current above permissible values may occur. To compensate this phenomenon, it is necessary to increase the power supply voltage or to shift the phase commutation cycle to the side of the lag, but this leads to an increase in the torque ripple.

\section{REFERENCES}

[1] Mounir Zeraoulia and Demba Diallo "Electric Motor Drive Selection Issues for HEV Propulsion Systems: A Comparative Study" IEEE Transactions on Vehicular Technology, Year: 2006, vol.: 55, Issue: 6, pp. 1756-1764.

[2] Zhi Yang, at al., "Comparative Study of Interior Permanent Magnet, Induction, and Switched Reluctance Motor Drives for EV and HEV Applications" IEEE Transactions on Transportation Electrification, vol. 1, no. 3, October 2015, pp. 245-254.

[3] R. M. Pindoriya, at al., "Comparative analysis of permanent magnet motors and switched reluctance motors capabilities for electric and hybrid electric vehicles" 2018 IEEMA Engineer Infinite Conference (eTechNxT), Year: 2018, pp. 1-5.

[4] V.P. Vujici'c, "Minimization of Torque Ripple and Copper Losses in Switched Reluctance Drive," IEEE Transactions on Power Electronics, vol. 27, no. 1, pp. 388-399, Jan. 2012.

[5] Russa K., Husain I., and Elbuluk M. "Torque ripple minimization in switched reluctance machines over a wide speed range" IEEE Trans. Ind. Applicat., vol. 34, pp. 1105-1112, Sept./Oct. 1998.

[6] D. S. Schramm, at al., "Torque ripple reduction of switched reluctance motors by phase current optimal profiling" in Proc. IEEE PESC'92, vol. 2, Toledo, Spain, 1992, pp. 857-860.

[7] Husain and M. Eshani, "Torque ripple minimization in switched reluctance motor drives by PWM current control," in Proc. IEEE PESC'94, vol. 1, 1994, pp. 72-77.

[8] Xue, X.D., at al., "Optimization and evaluation of torque-sharing functions for torque ripple minimization in switched reluctance motor drives" IEEE Trans. Power Electron., 2009, 24, (9), pp. 2076-2090.

[9] Jiayi Fan and Jin-Woo Ahn "Research on Direct Torque Control of Switched Reluctance Motor with Improved Commutation Strategy" 2018 IEEE International Conference on Industrial Technology (ICIT) Year: 2018.

[10] Qian Zhang, at al., "Torque Ripple Suppression of Switched Reluctance Motor Based on Torque Sharing Strategy," Advanced Materials Research, vol. 960, pp. 1086-1090, 2014.

[11] Tetsuya Kojima and Rik W. De Doncker "Optimal Torque Sharing in Direct Instantaneous Torque Control of Switched Reluctance Motors" 2015 IEEE Energy Conversion Congress and Exposition (ECCE), pp. 327-333.

[12] Krasovsky, A.B. and Bychkov, M.G. "An investigation of the ripples of the torque of a rectifier-type inductor electric drive". Elektrichestvo 2001 vol.10, pp. 33-43. (In Russian).

[13] Hui Zeng, Zhe Chen, and Hao Chen "Smooth Torque Speed Characteristic of Switched Reluctance Motors", Journal of Power Electronics, Vol. 14, No. 2, pp. 341-350, March 2014.

[14] Inderka R.B., De Doncker R.W. "DITC-Direct instantaneous torque control of switched reluctance drives" IEEE Transactions on Industry Applications, 2003. Vol. 39, № 4. pp. 1046-1051.

[15] Deepak Ronanki, Sheldon S Williamson "Comparative analysis of DITC and DTFC of switched reluctance motor for EV applications" 2017 IEEE International Conference on Industrial Technology (ICIT) Year: 2017 pp. 509-514.

[16] Hui Zeng, at al., "Direct instantaneous torque control with wide operating range for switched reluctance motors" IET Electric Power Applications, Year: 2015, Volume: 9, Issue: 9, pp.: 578-585.

[17] Miller T.J.E., "Electronic control of switched reluctance machines", Oxford: Newnes, 2001.

[18] Krishnan R., "Switched reluctance motor drives: modeling, simulation, analysis, design, and applications", Cambridge: CRC Press, 2001.

[19] Bychkov, at al.,. "Torque control of switched reluctance drive in generating mode" 2018 IEEE 25th International Workshop on Electric Drives: Optimization in Control of Electric Drives (IWED) Year: 2018.

[20] Krasovskii A.B. "The use of simulation to investigate a switched-reluctance electric drive", Elektrichestvo, 2003. No. 3, pp. 35-44.

[21] Miller T. J. E., McGilp M. "Nonlinear theory of the switched reluctance mo-tor for rapid computer-aided design". Proc. Inst. Elect. Eng., pt. B, vol. 137, № 6, Nov. 1990, pp. 337-347.

[22] Miller T. J. E., at al., "Ultra-fast model of the switched reluctance motor", in Conf. Rec. IEEE-IAS Annu. Meeting, St. Louis, MO, Oct. 1998, pp. 319-326. 


\section{BIOGRAPHIES OF AUTHORS}

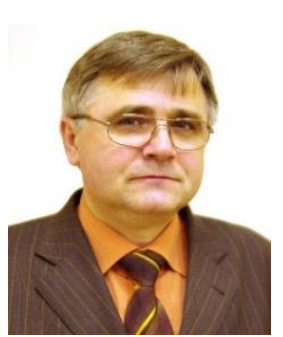

Alexander Krasovsky graduated from Moscow Power Engineering Institute in 1977. He received his Ph.D. and Dr.Sc. Degrees in electrical engineering from Moscow Power Engineering Institute in 1985 and 2004, respectively. In 2004, he was elected Professor for Dept. Electrical Engineering and Industrial Electronics Bauman Moscow State Technical University. Currently, he is Head of Department Electrical Engineering and Industrial Electronics Bauman Moscow State University. His main research interests include research and development of electric drives using modern computer technology. 